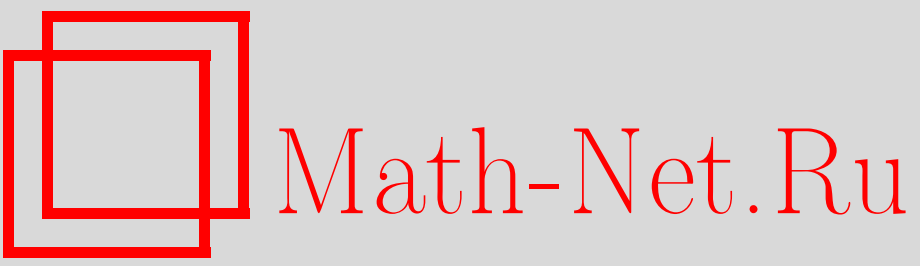

Ю. П. Чубурин, О спектре и собственных функциях двумерного оператора Шредингера с магнитным полем, ТМФ, 2003, том 134, номер 2, 243-253

DOI: https://doi.org/10.4213/tmf149

Использование Общероссийского математического портала Math-Net.Ru подразумевает, что вы прочитали и согласны с пользовательским соглашением

http://www.mathnet.ru/rus/agreement

Параметры загрузки:

IP: 3.85 .7 .115

26 апреля 2023 г., 12:29:06 
(C) 2003 г.

\author{
Ю.П. Чубурин*
}

\title{
О СПЕКТРЕ И СОБСТВЕННЫХ ФУНКЦИЯХ ДВУМЕРНОГО ОПЕРАТОРА ШРЕДИНГЕРА С МАГНИТНЫМ ПОЛЕМ
}

Доказан ряд теорем о собственных функциях и собственных значениях оператора Шредингера для большого постоянного магнитного поля.

Ключевые слова: оператор Шредингера, магнитное поле, собственная функция, собственное значение.

\section{1. ВВЕДЕНИЕ}

Рассмотрим оператор Шредингера

$$
H=H_{0}+V(x)
$$

действуюший в пространстве $L^{2}\left(\mathbb{R}^{2}\right)$, где

$$
H_{0}=-\frac{1}{2}\left(\left(\frac{\partial}{\partial x_{1}}-i B x_{2}\right)^{2}+\frac{\partial^{2}}{\partial x_{2}^{2}}\right)
$$

(выбрана калибровка Ландау), $B=$ const $>0$ - величина магнитного поля. Предполагаем, что потенциал $V(x)$ является вешественным и принадлежит $L^{2}\left(\mathbb{R}^{2}\right) \cap L^{\infty}\left(\mathbb{R}^{2}\right)$. Исследование такого рода операторов важно в связи с изучением эффекта Холла (см., например, [1]). Спектр $\sigma\left(H_{0}\right)$ оператора $H_{0}$, как известно, состоит из собственных значений бесконечной кратности (уровней Ландау) вида

$$
E_{n}=\left(n+\frac{1}{2}\right) B, \quad n=0,1, \ldots
$$

Из обших соображений легко вытекает включение $\sigma(H) \subset \bigcup_{n=0}^{\infty}\left[E_{n}-\|V\|_{\infty}, E_{n}+\right.$ $\left.\|V\|_{\infty}\right]$ (ср. [2]). Наконец, отметим, что существенный спектр $H$ совпадает с $\sigma\left(H_{0}\right)$ [3].

* Физико-технический институт УрО РАН, Ижевск, Россия. E-mail: chuburin@otf.fti.udmurtia.su 
Двумерные операторы Шредингера с магнитным полем изучались в ряде работ (см., например, [4]-[10]), но даже в случае убывающего потенциала и постоянного магнитного поля многие физически важные вопросы, связанные с местонахождением собственных значений и поведением собственных функций, остаются недостаточно исследованными. В данной работе получен ряд результатов о локализации собственных функций и собственных значений оператора $H$ для больших $B$ (или малых $V(x)$ ). В частности, в теореме 2 доказаны формулы, подобные формулам теории возмушений, но с остаточным членом, убывающим также при $B \rightarrow \infty$. Поясним в связи с этим, что теория возмущений для невозмущенного собственного значения бесконечной кратности теряет силу. В работе [4] получены обычные формулы теории возмущений для уровня бесконечной кратности, но для априорно взятых аналитических семейств собственных векторов и соответствующих собственных значений. В теореме 3 для случая $V(x)$ с компактным носителем доказана оценка для собственных функций двумерного магнитного оператора Шредингера, отвечающих собственным значениям, отделенным от уровня Ландау. Особенностью данной оценки, в отличие от известных [9], [10], является явное присутствие величины $B$ в константах. Это позволило доказать предельную (при $B \rightarrow \infty)$ локализацию собственных функций в круге, содержащем носитель $V(x)$ (см. следствие 2 и замечание 1). Результат, полученный в теореме 5, позволяет говорить о сгущении собственных значений при $B \rightarrow \infty$, что согласуется с результатами работ [7], [8] о плотности состояний. В теореме 6 доказано, что для потенциала с параметром вида $\mu V(x)$ сушественный спектр (в данном случае уровни Ландау) при $\mu \rightarrow \infty$ служит источником собственных значений. Аналогичный результат для другой разновидности оператора Шредингера можно найти, например, в статье [11].

В дальнейшем, чтобы подчеркнуть зависимость от магнитного поля, мы будем часто пользоваться обозначениями вида $H^{(B)}$ вместо $H$ и т.п. Норму в $L^{p}\left(\mathbb{R}^{2}\right)$ будем обозначать через $\|\cdot\|_{p}$.

\section{2. СОБСТВЕННЫЕ ФУНКЦИИ}

Обозначим через $P_{n}$ ортогональные проекторы на собственные подпространства $L_{n}=P_{n}\left(L^{2}\left(\mathbb{R}^{2}\right)\right)$ оператора $H_{0}$, отвечающие уровням Ландау $E_{n}=(n+1 / 2) B$. Имеем для резольвенты $R_{0}(E)=\left(H_{0}-E\right)^{-1}$ очевидное равенство

$$
R_{0}(E)=\sum_{n=0}^{\infty} \frac{P_{n}}{\left(n+\frac{1}{2}\right) B-E} .
$$

Введем обозначение $\rho\left(\psi, L_{n}\right)=\inf _{\phi \in L_{n}}\|\psi-\phi\|_{2}$.

ТеОрема 1. Виберем произвольно $\epsilon_{0}>0$. Пусть $\psi$-любой собственный вектор оператора $H^{(B)}$ такой, что $\|\psi\|_{2}=1$, отвечающий собственному значению $E \in$ $\left(E_{n_{0}}-B / 2, E_{n_{0}}+B / 2\right)$. Тогда имеет место равенство

$$
\rho\left(\psi, L_{n_{0}}\right)=O\left(\|V\|_{\infty} / B\right),
$$

причем $\left|O\left(\|V\|_{\infty} / B\right)\right| \leqslant\left(2+\epsilon_{0}\right)\|V\|_{\infty} / B$. 
ДокАЗАТЕльСтво. Перепишем равенство (1) в виде

$$
R_{0}(E)=\frac{P_{n_{0}}}{\left(n_{0}+\frac{1}{2}\right) B-E}+Q(E)
$$

где

$$
Q(E)=\sum_{n \neq n_{0}} \frac{P_{n}}{\left(n+\frac{1}{2}\right) B-E}
$$

- операторнозначная функция, аналитическая по аргументу $E$ в $(B / 2)$-окрестности точки $E_{n_{0}}$. Ее аналитичность вытекает из следуюшей равномерной по $E$ оценки:

$$
\|Q(E) \psi\|^{2}=\sum_{n \neq n_{0}} \frac{\left\|P_{n} \psi\right\|^{2}}{\left|\left(n+\frac{1}{2}\right) B-E\right|^{2}} \leqslant\left(\frac{2}{B}\right)^{2} \sum_{n \neq n_{0}}\left\|P_{n} \psi\right\|^{2} \leqslant\left(\frac{2}{B}\right)^{2}\|\psi\|^{2},
$$

где $\psi \in L^{2}\left(\mathbb{R}^{2}\right)$. Отсюда получаем

$$
\|Q(E)\| \leqslant \frac{2}{B}
$$

Выберем малое вешественное $\epsilon \neq 0$. Равенство $H \psi=E \psi$, где предполагается, что $\|\psi\|_{2}=1$, перепишем в виде

$$
\left(H_{0}-(E+\epsilon)\right) \psi=-(V+\epsilon) \psi .
$$

Последнее равенство для $E$ из $\epsilon$-окрестности точки $E_{n_{0}}$ с учетом соотношения $(2)$ эквивалентно равенству

$$
\psi=-R_{0}(E+\epsilon)(V+\epsilon) \psi=-\frac{P_{n_{0}}(V+\epsilon) \psi}{E_{n_{0}}-E-\epsilon}-Q(E+\epsilon)(V+\epsilon) \psi .
$$

Для тех собственных значений $E$, которые попадут в $\epsilon$-окрестность $E_{n_{0}}$, теорема вытекает из выражений (5), (4), если взять $\epsilon \leqslant \epsilon_{0}\|V\|_{\infty} / 2$. Для, возможно, оставшегося вне этой окрестности конечного числа собственных значений рассуждение аналогично, если положить $\epsilon=0$.

СлеДСтвИЕ 1. В условиях теоремы $\left\|\psi-P_{n_{0}}^{(B)} \psi\right\|=O\left(\|V\|_{\infty} / B\right)$.

ДоказАтельство. Сопоставим, пользуясь теоремой, вектору $\psi$ вектор $\psi_{0} \in$ $\operatorname{im} P_{n_{0}}^{(B)}$ такой, что $\left\|\psi-\psi_{0}\right\|=O\left(\|V\|_{\infty} / B\right)$. Тогда $\left\|\psi-P_{n_{0}}^{(B)} \psi\right\| \leqslant\left\|\psi-\psi_{0}\right\|+\| P_{n_{0}}^{(B)}\left(\psi_{0}-\right.$ $\psi) \|=O\left(\|V\|_{\infty} / B\right)$.

ТЕОрема 2. Пусть $\psi$ - собственный вектор оператора $H^{(B)}$ такой, что $\|\psi\|_{2}=1$, и отвечающий собственному значению $E \in\left(E_{n_{0}}-B / 2, E_{n_{0}}+B / 2\right)$, а $\psi_{0} \in \operatorname{im} P_{n_{0}}$ обладает тем свойством, ито

$$
\left\|\psi-\psi_{0}\right\|=O\left(\frac{\|V\|_{\infty}}{B}\right)
$$

(см. теорему 1). Тогда для $\Delta E=E-E_{n_{0}}$ имеют место равенства

$$
\Delta E=(V \psi, \psi)+O\left(\frac{\|V\|_{\infty}^{2}}{B}\right)=\left(V \psi_{0}, \psi_{0}\right)+O\left(\frac{\|V\|_{\infty}^{2}}{B}\right)
$$


ДокАЗАТЕЛЬСтво. Используя условие (6) и неравенство Шварца, выполним преобразование

$$
\begin{aligned}
\left(H \psi, \psi_{0}\right) & =E\left(\psi, \psi_{0}\right)=E(\psi, \psi)+O\left(\frac{\|V\|_{\infty}^{2}}{B}\right)=E+O\left(\frac{\|V\|_{\infty}^{2}}{B}\right)= \\
& =\left(\psi,\left(H_{0}+V\right) \psi_{0}\right)=E_{n_{0}}\left(\psi, \psi_{0}\right)+\left(\psi, V \psi_{0}\right) .
\end{aligned}
$$

Отсюда следует, что

$$
\begin{aligned}
\left(E-E_{n_{0}}\right)\left(\psi, \psi_{0}\right) & =\Delta E+O\left(\frac{\|V\|_{\infty}^{2}}{B}\right)=\left(\psi, V \psi_{0}\right)= \\
& =\left(\psi_{0}, V \psi_{0}\right)+O\left(\frac{\|V\|_{\infty}^{2}}{B}\right)=(\psi, V \psi)+O\left(\frac{\|V\|_{\infty}^{2}}{B}\right) .
\end{aligned}
$$

Полученные соотношения доказьвают теорему.

Введем обозначение $|x|=\sqrt{x_{1}^{2}+x_{2}^{2}}$.

ТЕОРЕма 3. Предположим, что для носителя $V$ справедливо включение $\operatorname{supp} V \subset\left\{x \in \mathbb{R}^{2}:|x| \leqslant R\right\}$. Пусть $\epsilon, \delta>0$. Существуют такие константь $C$, $k>0$, что для произвольной собственной функции $\psi(x)$ оператора $H^{(B)}$ такой, что $\|\psi\|=1$, а соответствующее собственное значение $E$ удовлетворяет условию $B / 2>\left|E-E_{n_{0}}\right| \geqslant \epsilon$, и для всех $x$ таких, что $|x| \geqslant R+\delta$, справедлива оченка

$$
|\psi(x)| \leqslant C\|V\|_{\infty} B e^{-k B x^{2}} .
$$

ДокаЗАТЕльство. Оценим функцию Грина (ядро резольвенты) $G_{0}^{(B)}(x, y, E)$ оператора $H_{0}^{(B)}$. Согласно [12] выбор калибровки приводит к переходу от оператора $H$ к оператору $e^{-i \lambda(x)} H e^{i \lambda(x)}$, где $\lambda(x)$ - некоторая вещественная функция. Поэтому можно воспользоваться при оценке видом функции Грина в симметричной калибровке [5]:

$$
\begin{aligned}
G_{0}^{(B)}(x, y, E)= & \frac{1}{2 \pi} \Gamma\left(\frac{1}{2}-\frac{E}{B}\right) e^{-i B(x \wedge y) / 2} \cdot e^{-B(x-y)^{2} / 4} \times \\
& \times \Psi\left(\frac{1}{2}-\frac{E}{B}, 1, \frac{B(x-y)^{2}}{2}\right)
\end{aligned}
$$

где $\Gamma$ - гамма-функция, $x \wedge y=x_{1} y_{2}-x_{2} y_{1}, \quad \Psi$ - вырожденная гипергеометрическая функция второго рода. Имеем неравенства

$$
\left(n_{0}+\frac{1}{2}\right) B-\|V\|_{\infty}<E<\left(n_{0}+\frac{1}{2}\right) B+\|V\|_{\infty},
$$

откуда

$$
-n_{0}+\frac{\|V\|_{\infty}}{B}>\frac{1}{2}-\frac{E}{B}>-n_{0}-\frac{\|V\|_{\infty}}{B} .
$$


Следовательно, для достаточно больших $B$ величина $(1 / 2)-(E / B)$ находится в сколь угодно малой окрестности точки $-n_{0}$. Далее, функция $\Gamma$ имеет полюс первого порядка в точке $-n_{0}$. С использованием условия теоремы для достаточно больших $B$ получаем

$$
\left|\Gamma\left(\frac{1}{2}-\frac{E}{B}\right)\right| \leqslant \frac{C}{\left|\frac{1}{2}-\frac{E}{B}+n_{0}\right|}=\frac{C B}{\left|E_{n_{0}}-E\right|} \leqslant C_{1} B
$$

Наконец, заметим, что согласно [13]

$$
\Psi(\alpha, 1, z)=\frac{1}{z^{\alpha}}+O\left(\frac{1}{z^{\alpha+1}}\right)
$$

где $-\pi<\arg z \leqslant \pi, z \neq 0$.

Пусть $\psi(x)$ - собственная функция оператора $H^{(B)}$, удовлетворяюшая условию теоремы. Имеем

$$
\psi(x)=-\int_{\mathbb{R}^{2}} G_{0}^{(B)}(x, y, E) V(y) \psi(y) d y,
$$

откуда

$$
|\psi(x)| \leqslant\left(\int_{\mathbb{R}^{2}}\left|G_{0}^{(B)}(x, y, E)\right|^{2}|V(y)|^{2} d y\right)^{1 / 2} .
$$

Предположим, что $|y| \leqslant R,|x| \geqslant R+\delta$, тогда для достаточно больших $B$ имеет место неравенство $B(x-y)^{2} / 2 \geqslant 1$ (действительно, в противном случае $|x| \leqslant|x-y|+|y|<$ $\sqrt{2 / B}+R<R+\delta$ для больших $B)$. Пусть $\sigma \in(0,1)$ произвольно. Оценим $|\psi(x)|^{2}$ для достаточно больших $B$ и $|x| \geqslant R+\delta$, пользуясь проведенными выше рассуждениями и соотношениями (7)-(11):

$$
\begin{aligned}
|\psi(x)|^{2} \leqslant & \int_{\left\{B(x-y)^{2} / 2 \geqslant 1\right\}}\left|G_{0}^{(B)}(x, y, E)\right|^{2}|V(y)|^{2} d y \leqslant \\
\leqslant & C B^{2}\|V\|_{\infty}^{2} \int_{\left\{B(x-y)^{2} / 2 \geqslant 1\right\} \cap\{|y| \leqslant R\}}\left(\frac{1}{2} B(x-y)^{2}\right)^{2(E / B-1 / 2)} \times \\
& \times e^{-B(x-y)^{2} / 2} d y \leqslant C_{1} B^{2}\|V\|_{\infty}^{2} \int_{\{|y| \leqslant R\}} \exp \left[-\frac{1}{2}(1-\sigma) B(x-y)^{2}\right] d y \leqslant \\
\leqslant & C_{1} B^{2}\|V\|_{\infty}^{2} \int_{\{|y| \leqslant R\}} \exp \left[-\frac{1}{2}(1-\sigma) B|| x|-| y||^{2}\right] d y= \\
= & C_{1} B^{2}\|V\|_{\infty}^{2} \int_{\{|y| \leqslant R\}} \exp \left[-\frac{1}{2}(1-\sigma) B|x|^{2}\left|1-\frac{|y|}{|x|}\right|^{2}\right] d y \leqslant \\
\leqslant & C_{1} B^{2}\|V\|_{\infty}^{2} \exp \left[-\frac{1}{2}(1-\sigma) B \delta^{2}(R+\delta)^{-2}|x|^{2}\right] \pi R^{2} .
\end{aligned}
$$

Полученное неравенство и доказывает теорему. 
СЛЕДСТВИЕ 2. В условиях теоремы имеет место неравенство

$$
\int_{\{|x| \geqslant R+\delta\}}|\psi(x)|^{2} d x \leqslant C e^{-a B}, \quad a>0
$$

ДокАЗАТЕЛЬСтво. Переходя к полярным координатам, получаем оценку

$$
\int_{\{|x| \geqslant R+\delta\}}|\psi(x)|^{2} d x \leqslant C B^{2} \int_{R+\delta}^{\infty} e^{-2 k B \rho^{2}} \rho d \rho=\frac{C B}{4 k} e^{-2 k B(R+\delta)^{2}} \leqslant C_{1} e^{-a B}
$$

где $a \in\left(0,2 k(R+\delta)^{2}\right)$ произвольно.

ЗАМЕчАнИЕ 1 . В случае если $V(x) \geqslant 0$ или $V(x) \leqslant 0$ для всех $x$, из следствия 2 вытекает, что при $B \rightarrow \infty$ и $E \rightarrow E_{n_{0}}$

$$
\int_{\left\{x:|V(x)| \geqslant \epsilon_{0}>0\right\}}|\psi(x)|^{2} d x \rightarrow 0
$$

где $\epsilon_{0}$ - любое положительное число. Это означает, что состояния частицы с данными энергиями $E$ в пределе локализуются вне "области локализации” потенциала. Если же $B \rightarrow \infty$, но энергии $E$, наоборот, отделены от $E_{n_{0}}$, то в случае финитного потенциала согласно следствию 2 состояния частицы стремятся локализоваться там же, где сосредоточен потенциал.

\section{3. СОБСТВЕННЫЕ ЗНАЧЕНИЯ}

В данном разделе будет предполагаться, что потенциал $V(x)$ ненулевой и знакопостоянный для всех $x$, т.е. $V(x) \geqslant 0$ (или $V(x) \leqslant 0$ ).

ЛЕмма 1 (по поводу доказательства см. [14]). Пусть $A$ - самосопряженный оператор в гильбертовом пространстве $\mathcal{H}$ и для некоторых $\phi, \psi \in \mathcal{H} u \lambda \in \mathbf{C}$ таких, что $\|\phi\|=1,\|\psi\|=\epsilon$, выполнено равенство $А \phi=\lambda \phi+\psi$. Тогда

$$
\rho(\lambda, \sigma(A))=\inf _{\mu \in \sigma(A)}|\lambda-\mu| \leqslant \epsilon
$$

Заметим, что условие $V \in L^{2}\left(\mathbb{R}^{2}\right) \cap L^{\infty}\left(\mathbb{R}^{2}\right)$ обеспечивает квадратичную суммируемость ядер операторов $\sqrt{V} P_{n} \sqrt{V}$ и $P_{n} V$. Это вытекает из вида ядра $P_{n}$ в симметричной калибровке [5](калибровка здесь роли не играет, см. доказательство теоремы 3 ). Аналогично ядра операторов $\sqrt{V} R_{0}(E) \sqrt{V}$ и $R_{0}(E) V$ тоже квадратично-суммируемы. Это следует из вида ядра $R_{0}(E)$ и известных асимптотических свойств вырожденной гипергеометрической функции второго рода. Таким образом, все перечисленные операторы компактны. 
ТЕОрема 4. Пусть $\epsilon_{0}>0$. Для любого собственного значения $E \in\left(E_{n_{0}}-B / 2\right.$, $\left.E_{n_{0}}+B / 2\right) \backslash\left\{E_{n_{0}}\right\}$ оператора $H$ найдется собственное значение $\lambda$ оператора $\sqrt{V} P_{n_{0}} \sqrt{V}$ (а также для достаточно мальх $\|V\|_{\infty} / B$ для любого собственного значения $\lambda \neq 0$ оператора $\sqrt{V} P_{n_{0}} \sqrt{V}$ найдется собственное значение $E$ оператора $H$ ) такое, что имеет место равенство

$$
E=E_{n_{0}}+\lambda+O\left(\frac{\|V\|_{\infty}^{2}}{B}\right)
$$

причем

$$
\left|O\left(\frac{\|V\|_{\infty}^{2}}{B}\right)\right| \leqslant\left(2+\epsilon_{0}\right) \frac{\|V\|_{\infty}^{2}}{B} .
$$

ДокАЗАтЕльство. Уравнение Шредингера $H \psi=E \psi$ для рассматриваемых $E$ можно переписать в виде уравнения $\psi=-R_{0}(E) V \psi$ или, после известной замены $\phi=\sqrt{V} \psi$ [15], в виде (см. (2))

$$
\phi=-\sqrt{V} R_{0}(E) \sqrt{V} \phi=-\frac{\sqrt{V} P_{n_{0}} \sqrt{V} \phi}{E_{n_{0}}-E}-\sqrt{V} Q(E) \sqrt{V} \phi .
$$

При этом операторы $\sqrt{V} R_{0}(E) \sqrt{V}, \sqrt{V} P_{n_{0}} \sqrt{V}$ и $\sqrt{V} Q(E) \sqrt{V}$ являются самосопряженными в силу знакопостоянства $V$.

Пусть $E \in\left(E_{n_{0}}-B / 2, E_{n_{0}}+B / 2\right) \backslash\left\{E_{n_{0}}\right\}$, тогда для некоторого $\phi \in L^{2}\left(\mathbb{R}^{2}\right)$ с единичной нормой выполнено уравнение (12). Используя лемму 1 и оценку (4), получаем неравенство

$$
\rho\left(E-E_{n_{0}}, \sigma\left(\sqrt{V} P_{n_{0}} \sqrt{V}\right)\right) \leqslant \frac{2\|V\|_{\infty}\left|E-E_{n_{0}}\right|}{B} \leqslant \frac{2\|V\|_{\infty}^{2}}{B},
$$

из которого и вытекает первое утверждение теоремы.

Пусть, наоборот, $\lambda \in \sigma\left(\sqrt{V} P_{n_{0}} \sqrt{V}\right) \backslash\{0\}, \phi$ - соответствующий собственный вектор такой, что $\|\phi\|_{2}=1$. Будем предполагать в соответствии с условием теоремы, что $\|V\|_{\infty} / B<1 / 2$. Положим $E=E_{n_{0}}+\lambda$, тогда

$$
\left|E-E_{n_{0}}\right|=|\lambda|=\left\|\sqrt{V} P_{n_{0}} \sqrt{V} \phi\right\|_{2} \leqslant\|V\|_{\infty}<\frac{B}{2} .
$$

Вследствие (2)

$$
\sqrt{V} R_{n_{0}}(E) \sqrt{V} \phi=-\phi+\sqrt{V} Q(E) \sqrt{V} \phi,
$$

откуда, как и выше, следует, что

$$
\rho\left(-1, \sigma\left(\sqrt{V} R_{n_{0}}(E) \sqrt{V}\right)\right) \leqslant \frac{2\|V\|_{\infty}}{B} .
$$

Таким образом, для любого $\epsilon>0$ сушествует собственное значение $\mu$ оператора $\sqrt{V} R_{n_{0}}(E) \sqrt{V}$, для которого

$$
|\mu+1| \leqslant \frac{(2+\epsilon)\|V\|_{\infty}}{B} .
$$


Пусть $\|V\|_{\infty} / B$ настолько мало, что $\mu<0$ и $|\mu| \geqslant 1-\delta>0$ для некоторого $\delta>0$. Число -1 , очевидно, является собственным значением оператора $\sqrt{V /|\mu|} R_{n_{0}}(E) \sqrt{V /|\mu|}$. Следовательно, $E$ - собственное значение оператора $H_{0}+V /|\mu|$. Имеем в силу (13)

$$
\left|V(x)-\frac{V(x)}{|\mu|}\right|=|V(x)|\left|\frac{1+\mu}{\mu}\right| \leqslant \frac{(2+\epsilon)\|V\|_{\infty}^{2}}{B(1-\delta)} .
$$

Уравнение Шредингера $\left(H_{0}+|\mu|^{-1} V\right) \psi=E \psi$ перепишем в виде

$$
\left(H_{0}+V\right) \psi=E \psi+V\left(1-|\mu|^{-1}\right) \psi
$$

В силу сказанного выше сушествует решение данного уравнения такое, что $\|\psi\|_{2}=1$. При этом из леммы 1 и выражения (14) для достаточно малых $\epsilon, \delta$ вытекает неравенство

$$
\rho(E, \sigma(H))=\rho\left(E_{n_{0}}+\lambda, \sigma(H)\right)<\frac{\left(2+\epsilon_{0}\right)\|V\|_{\infty}^{2}}{B},
$$

что и доказывает второе утверждение теоремы.

ЗАмЕчАнИЕ 2. Из (12) после скалярного умножения равенства на $\varnothing$ легко выводится, что если $\|V\|_{\infty}<B / 2$, то собственные значения оператора $H$ из $\left(E_{n_{0}}-B / 2, E_{n_{0}}+\right.$ $B / 2$ ) лежат левее или правее $E_{n_{0}}$ в зависимости от знака $V(x)$ (для $n_{0}=0$ это тривиально).

ЗАмечание 3 . Пусть $E=E_{n_{0}}$ - собственное значение оператора $H, \psi$ - соответствуюшая собственная функция. Тогда $\lambda=0$ - собственное значение оператора $P_{n_{0}} V$, отвечающее этой же собственной функции (если $\sqrt{V} \psi \neq 0$, то $\lambda=0$ - собственное значение также и оператора $\left.\sqrt{V} P_{n_{0}} \sqrt{V}\right)$. Действительно, действуя на обе части равенства $\left(H_{0}+V\right) \psi=E_{n_{0}} \psi$ оператором $P_{n_{0}}$, получаем $P_{n_{0}} V \psi=0$.

Обозначим через $A \subset \mathbb{R}^{2}$ совокупность точек, в которых функция $V(x)$ непрерывна. Положим $V(A)=\{V(x): x \in A\}$.

Теорема 5. Для любой последовательности $B_{m} \rightarrow \infty \quad(m \rightarrow \infty)$ и для любого $\lambda \in V(A)$ существует последовательность собственных значений $E_{m}^{\left(n_{0}\right)} \in$ $\sigma\left(H^{\left(B_{m}\right)}\right) \operatorname{maxux,~umo~} \lim _{m \rightarrow \infty} E_{m}^{\left(n_{0}\right)}=E_{n_{0}}+\lambda$.

ДокАЗАТЕЛЬСТво. В силу теоремы 4 достаточно доказать для $\lambda \neq 0$ сушествование последовательности собственных значений $\lambda_{m} \in \sigma\left(\sqrt{V} P_{n_{0}}^{\left(B_{m}\right)} \sqrt{V}\right)$ таких, что $\lim _{m \rightarrow \infty} \lambda_{m}=\lambda$. В случае $\lambda=0$ требуемая последовательность $\left\{E_{m}^{\left(n_{0}\right)}\right\}$ всегда найдется, так как сушественный спектр оператора $H$ совпадает со спектром $H_{0}[3]$. Поскольку сдвиг не меняет спектра оператора $H[5]$, то можно считать, что $\lambda=V(0)$. Выясним поведение собственных значений оператора $\sqrt{V} P_{n_{0}}^{(B)} \sqrt{V}$ при $B \rightarrow \infty$. Для $\phi \in L^{2}\left(\mathbb{R}^{2}\right)$ имеем равенство

$$
\sqrt{V} P_{n_{0}}^{(B)} \sqrt{V} \phi=B \int_{\mathbb{R}^{2}} \sqrt{V(x)} K_{n_{0}}^{(1)}(\sqrt{B} x, \sqrt{B} y) \sqrt{V(y)} \phi(y) d y
$$


где $K_{n_{0}}^{(B)}(x, y)$ - ядро оператора $P_{n_{0}}^{(B)}[5]$. Определим в $L^{2}\left(\mathbb{R}^{2}\right)$ унитарный оператор форомулой

$$
U^{(B)} \phi(x)=B^{-1 / 2} \phi\left(B^{-1 / 2} x\right) .
$$

Из равенства (15) вытекает, что $U^{(B)}$ устанавливает унитарную эквивалентность операторов $\sqrt{V(x)} P_{n_{0}}^{(B)} \sqrt{V(x)}$ и $\sqrt{V(x / \sqrt{B})} P_{n_{0}}^{(1)} \sqrt{V(x / \sqrt{B})}$. Пусть $\phi \in C_{0}^{\infty}\left(\mathbb{R}^{2}\right)$. В силу теоремы Лебега о предельном переходе для всех $x \in \mathbb{R}^{2}$ имеем при $B=B_{m} \rightarrow \infty$ :

$$
\begin{aligned}
& \sqrt{V \frac{x}{\sqrt{B}}} P_{n_{0}}^{(1)} \sqrt{V \frac{x}{\sqrt{B}}} \phi(x)=\int_{\mathbb{R}^{2}} \sqrt{V \frac{x}{\sqrt{B}}} K_{n_{0}}^{(1)}(x, y) \sqrt{V \frac{y}{\sqrt{B}}} \phi(y) d y \rightarrow \\
& \rightarrow V(0) \int_{\mathbb{R}^{2}} K_{n_{0}}^{(1)}(x, y) \phi(y) d y=V(0) P_{n_{0}}^{(1)} \phi(x) .
\end{aligned}
$$

При этом (см. вид функции $K_{n}^{(1)}$ в работе [5])

$$
\begin{aligned}
& \left|\int_{\mathbb{R}^{2}} \sqrt{V \frac{x}{\sqrt{B}}} K_{n_{0}}^{(1)}(x, y) \sqrt{V \frac{y}{\sqrt{B}}} \phi(y) d y\right| \leqslant \\
& \quad \leqslant \frac{\|V\|_{\infty}}{2 \pi} \int_{\mathbb{R}^{2}} e^{-(x-y)^{2} / 4}\left|L_{n_{0}} \frac{(x-y)^{2}}{2}\right||\phi(y)| d y,
\end{aligned}
$$

где $L_{n_{0}}$ - многочлен Лагерра. Интеграл в правой части (17) представляет собой свертку функций из $L^{1}\left(\mathbb{R}^{2}\right)$ и $L^{2}\left(\mathbb{R}^{2}\right)$ и в силу неравенства Юнга [16] принадлежит $L^{2}\left(\mathbb{R}^{2}\right)$. Опять применяя теорему Лебега, получаем, что сходимость в (16) имеет место в $L^{2}\left(\mathbb{R}^{2}\right)$. По теореме Банаха-Штейнхауза операторы $\sqrt{V(x / \sqrt{B})} P_{n_{0}}^{(1)} \sqrt{V(x / \sqrt{B})}$ сходятся поточечно к оператору $V(0) P_{n_{0}}^{(1)}$ при $B=B_{m} \rightarrow \infty$. Вследствие теоремы VIII.25 из книги [17] имеет место сильная резольвентная сходимость этих операторов. Спектр оператора $V(0) P_{n_{0}}^{(1)}$ состоит из чисел 0 и $V(0)$. Согласно теореме VIII.24 из той же книги сушествуют собственные значения

$$
\begin{gathered}
\lambda_{m} \in \sigma\left(\sqrt{V\left(x / \sqrt{B_{m}}\right)} P_{n_{0}}^{(1)} \sqrt{V\left(x / \sqrt{B_{m}}\right)}\right)=\sigma\left(\sqrt{V(x)} P_{n_{0}}^{\left(B_{m}\right)} \sqrt{V(x)}\right), \\
m=1,2, \ldots,
\end{gathered}
$$

такие, что $\lim _{m \rightarrow \infty} \lambda_{m}=V(0)$. Теорема доказана.

ЗАМЕЧАНИЕ 4. Из результатов работ [7], [8] о плотности состояний вытекает, что в условиях теоремы 5 для каждой точки $\lambda \in V(A)$ найдется, вообше говоря, своя последовательность $B_{m}=B_{m}(\lambda) \rightarrow \infty, m \rightarrow \infty$, для которой справедливо утверждение теоремы.

Положим $H(\mu)=H_{0}+\mu V(x), \mu \in \mathbb{R}\left(\mu-\right.$ "константа связи"). Если $E=E\left(\mu_{0}\right) \neq$ $E_{n_{0}}$ - собственное значение оператора $H\left(\mu_{0}\right)$ кратности $N$, то в окрестности $\mu_{0}$ собственные значения $E$, находяшиеся вблизи $E_{n_{0}}$, задаются $N$ аналитическими функциями $E=E_{i}(\mu), i=1, \ldots, N$ (см. [18], добавление 2, теорема 3.1 ). 
ТеОрема 6. (ср. [11], раздел 3). Предположим, что $V(x)$ не обращается в нуль и для некоторого $N>0$ справедлива оценка $|V(x)|^{-1} \leqslant(1+|x|)^{N}$. Пусть $E \in\left(E_{n_{0}}-\right.$ $\left.B / 2, E_{n_{0}}+B / 2\right), \quad E \neq E_{n_{0}} u \operatorname{sign}\left(E-E_{n_{0}}\right)=\operatorname{sign} V$. Предположим, что величина $\left|E-E_{n_{0}}\right|$ достаточно мала или $B$ достаточно велико. Тогда существуют такие $\mu_{m}>0, \quad m=1,2, \ldots$, что $\sum_{m=1}^{\infty} \mu_{m}^{-2}<\infty u E \in \sigma\left(H\left(\mu_{m}\right)\right)$. При этом функиии $E_{i}(\mu), \quad i=1, \ldots, N$, с возрастанием $\mu$ переходят точку $E$ при $\mu=\mu_{m}$, $m=1,2, \ldots$, справа налево, если $V \leqslant 0$, и слева направо, если $V \geqslant 0$.

ДокАЗАтЕльство. Оператор $\sqrt{V} R_{0}(E) \sqrt{V}$ самосопряжен и ограничен. В силу леммы Глазмана ([18], добавление 1$)$ необходимым и достаточным условием наличия у такого оператора бесконечного множества отрицательных собственных значений является сушествование бесконечномерного линейного многообразия $M \subset L^{2}\left(\mathbb{R}^{2}\right)$ такого, что для $\psi \in M \backslash\{0\}$ справедливо неравенство $\left(\sqrt{V} R_{0}(E) \sqrt{V} \psi, \psi\right)<0$.

Докажем вначале, что линейное многообразие $L=\operatorname{im} P_{n_{0}} \cap \mathcal{S}\left(\mathbb{R}^{2}\right)$, где $\mathcal{S}\left(\mathbb{R}^{2}\right)$ - пространство быстроубываюших функций, бесконечномерно. Уравнение Шредингера $H_{0} \psi=E \psi$ после преобразования Фурье по переменной $x_{1}$ приводится к уравнению для собственных значений для гармонического осциллятора (см., например, [7], [8]). Пользуясь этим, легко усмотреть, что функции вида

$$
\psi=\int_{\mathbb{R}} e^{i x_{1} p_{1}} C\left(p_{1}\right) \exp \left[-\frac{1}{2}\left(\sqrt{B} x_{2}-\frac{p_{1}}{\sqrt{B}}\right)^{2}\right] H_{n_{0}}\left(\sqrt{B} x_{2}-\frac{p_{1}}{\sqrt{B}}\right) d p_{1},
$$

где $H_{n_{0}}(x)$ - полином Эрмита, а $C\left(p_{1}\right) \in L^{2}(\mathbb{R})$, принадлежат $\operatorname{im} P_{n_{0}}$ (константы в преобразовании Фурье выбираем как в [16], раздел IX.2). Без труда доказывается, что в случае $C\left(p_{1}\right) \in \mathcal{S}(\mathbb{R})$ имеет место включение $\psi(x) \in \mathcal{S}\left(\mathbb{R}^{2}\right)$.

Выбирая бесконечную последовательность линейно независимых функций $C(k)=$ $C_{\nu}(k), \nu=1,2, \ldots$, получаем линейную независимость соответствующих собственных функций $\psi=\psi_{\nu}(x)$. Следовательно, линейное многообразие $L$ бесконечномерно. Поэтому бесконечномерным будет и линейное многообразие $M=\{\phi / \sqrt{V}: \phi \in L\} \subset L^{2}\left(\mathbb{R}^{2}\right)$ (последнее включение выполнено в силу условия теоремы).

Пусть $\psi=\phi / \sqrt{V} \in M \backslash\{0\}$, где $\phi \in L$. Считаем для определенности, что $V<0$. Если $E_{n_{0}}-E$ или $B^{-1}$ мало, для всех $\psi \in M \backslash\{0\}$, пользуясь выражениями (2) и (4), выполним следующее преобразование:

$$
\begin{aligned}
& \left(\sqrt{V} R_{0}(E) \sqrt{V} \psi, \psi\right)=-\left(R_{0}(E) \phi, \phi\right)=-\frac{\left(P_{n_{0}} \phi, \phi\right)}{E_{n_{0}}-E}-(Q(E) \phi, \phi)= \\
& \quad=\|\phi\|^{2}\left(-\frac{(\phi /\|\phi\|, \phi /\|\phi\|)}{E_{n_{0}}-E}-(Q(E)(\phi /\|\phi\|), \phi /\|\phi\|)\right)= \\
& \quad=\|\phi\|^{2}\left(-\frac{1}{E_{n_{0}}-E}+O\left(B^{-1}\right)\right)<0 .
\end{aligned}
$$

Таким образом, у оператора $\sqrt{V} R_{0}(E) \sqrt{V}$ имеется бесконечное число отрицательных собственных значений $\lambda_{1}<\cdots<\lambda_{n}<\cdots<0$. Так как оператор $\sqrt{V} R_{0}(E) \sqrt{V}$ является оператором Гильберта-Шмидта, то $\sum_{n=1}^{\infty} \lambda_{n}^{2}<\infty$. Положим $\mu_{n}=\left|\lambda_{n}\right|^{-1}$, тогда 
оператор

$$
\mu_{n} \sqrt{V} R_{0}(E) \sqrt{V}=\sqrt{\mu_{n} V} R_{0}(E) \sqrt{\mu_{n} V}
$$

имеет собственное значение, равное - 1 , а следовательно, $E \in \sigma\left(H\left(\mu_{n}\right)\right)$ (см. доказательство теоремы 4). Из сказанного вытекает первое утверждение теоремы.

Для каждой из аналитических функций $E_{i}(\mu)$ (см. замечание 4) из формулы Рэлея ([18], приложение 1) вытекает соотношение

$$
\frac{d E_{i}(\mu)}{d \mu}=\left(V \psi_{i}(x, \mu), \psi_{i}(x, \mu)\right)<0
$$

где через $\psi_{i}(x, \mu)$ обозначена собственная функция оператора $H(\mu)$, по норме равная единище, отвечающая собственному значению $E_{i}(\mu)$. Теорема доказана.

\section{Список литературы}

[1] S. M. Girvin, R. E. Pranger (Eds). The Quantum Hall Effect. Berlin: Springer, 1987.

[2] М. М. Скриганов. Тр. МИАН. 1985. Т. 171. С. 1.

[3] A. Iwatsuka. J. Math. Kyoto Univ. 1983. V. 23. P. 475.

[4] А.С. Льскова. ТМФ. 1985. Т. 65. № 3. С. 368.

[5] В. А. Гейлер. Алгебра и анализ. 1991. Т. 3. № 3. С. 1.

[6] H. Grosse. Lett. Math. Phys. 1995. V. 34. P. 59.

[7] G. D. Raikov. Commun. Part. Diff. Equat. 1998. V. 23. P. 1583.

[8] M. Dimassi, G. D. Raikov. Spectral asymptotics for quantum Hamiltonians in strong magnetic fields. Preprint mp-arc 01-294. Texas Math. Physics archive, 2001.

[9] H. D. Cornean, G. Nenciu. Commun. Math. Phys. 1998. V. 192. P. 671

[10] H. D. Cornean, G. Nenciu. Two-dimensional magnetic schrödinger operators: width of minibands in the tight binding approximation. Preprint E.S.I. № 608. Vienna, 1998.

[11] Ю. П. Чубурин. ТМФ. 2001. Т. 126. С. 196.

[12] Х. Цикон, Р. Фрезе, В. Кири, Б. Саймон. Операторы Шредингера с приложениями к квантовой механике и глобальной геометрии. М.: Мир, 1990.

[13] А. Ф. Никифоров, В. Б. Уваров. Специальные функции математической физики. М.: Наука, 1984.

[14] В. Ф. Лазуткин. Квазиклассическая асимптотика собственных функций. В сб.: Итоги науки и техники. Современные проблемы математики. Фундаментальные направления. Т. 34. Дифференциальные уравнения с частными производными-5. Ред. Р.В. Гамкрелидзе. М.: ВИНИТИ, 1988. C. 135.

[15] М. Рид, Б. Саймон. Методы современной математической физики. Т. 3. Теория рассеяния. М.: Мир, 1982.

[16] М. Рид, Б. Саймон. Методы современной математической физики. Т. 2. Гармонический анализ. Самосопряженность. М.: Мир, 1978.

[17] М. Рид, Б. Саймон. Методы современной математической физики. Т. 1. Функциональный анализ. М.: Мир, 1977.

[18] Ф. А. Березин, М. А. Шубин. Уравнение Шредингера. М.: Изд-во МГУ, 1983.

Поступила в редакцию 11.I.2002 г., после доработки 17.VII.2002 г. 